\title{
Characterization of highly proliferative secondary tumor clusters along host blood vessels in malignant glioma
}

\author{
TING-CHUNG WANG ${ }^{1,2}$, CHUN-YU CHENG $^{1}$, WEI-HSUN YANG ${ }^{1,2}$, WEN-CHENG CHEN $^{3}$ and PEY-JIUM CHANG ${ }^{2}$ \\ ${ }^{1}$ Department of Neurosurgery, Chang-Gung Memorial Hospital, Chiayi 613; \\ ${ }^{2}$ Graduate Institute of Clinical Medical Sciences, Chiayi Branch, College of Medicine, Chang-Gung University, Chiayi 613; \\ ${ }^{3}$ Department of Radiation Oncology, Chang-Gung Memorial Hospital, Chiayi 613, Taiwan, R.O.C.
}

Received August 14, 2014; Accepted June 15, 2015

DOI: $10.3892 / \mathrm{mmr} .2015 .4228$

\begin{abstract}
The aim of the present study was to investigate the extensive invasion of tumor cells into normal brain tissue, a life-threatening feature of malignant gliomas. How invasive tumor cells migrate into normal brain tissue and form a secondary tumor structure remains to be elucidated. In the present study, the morphological and phenotypic changes of glioma cells during invasion in a C6 glioma model were investigated. C6 glioma cells were stereotactically injected into the right putamen region of adult Sprague-Dawley rats. The brain tissue sections were then subjected to hematoxylin and eosin, immunohistochemical or immunofluorescent staining. High magnification views of the tissue sections revealed that C6 cells formed tumor spheroids following implantation and marked invasion was observed shortly after spheroid formation. In the later stages of invasion, certain tumor cells invaded the perivascular space and formed small tumor clusters. These small tumor clusters exhibited certain common features, including tumor cell multilayers surrounding an arteriole, which occurred up to several millimeters away from the primary tumor mass; a high proliferation rate; and similar gene expression profiles to the primary tumor. In conclusion, the present study revealed that invading tumor cells are capable of forming highly proliferative cell clusters along arterioles near the tumor margin, which may be a possible cause of the recurrence of malignant glioma.
\end{abstract}

\section{Introduction}

Glioblastoma multiforme (GBM) is the most common and aggressive type of glioma with an extremely poor prognosis. Patients with GBM have an average survival time of 1-2 years

Correspondence to: Dr Pey-Jium Chang, Graduate Institute of Clinical Medical Sciences, Chiayi Branch, College of Medicine, Chang-Gung University, 6 West, Chia-Pu Road, Puzi, Chiayi 613, Taiwan, R.O.C.

E-mail: peyjiumc@mail.cgu.edu.tw

Key words: C6 glioma, perivascular invasion, secondary tumor cluster after treatment with the available therapies (1). This poor prognosis is presumed to be due to its invasive nature, as the majority of patients with GBM die from local recurrence, which usually occurs within $2-3 \mathrm{~cm}$ of the resection margin (2). Thus, understanding the phenotypic characteristics and biochemical features of invasive tumor cells may be critical in the development of a potential treatment for this incurable condition.

Numerous experimental animal models, which share the characteristics of human GBM have been developed via the intracranial implantation of malignant glioma cell lines into rodents. Among these glioma models, the C6 rat glioma cell line represents an important and widely used model, which is easily handled and maintained. Although different implantation protocols $(3,4)$ or rodent hosts $(5,6)$ used to develop the model may affect the overall period of tumor progression, the key steps in tumorigenesis, including tumor growth, angiogenesis and invasion, are generally similar (7).

Following implantation of C6 cells in rodent hosts, C6 cells initially grow as a tumor spheroid and then certain C6 cells aggressively invade into the normal brain tissue along white matter tracts or blood vessels $(8,9)$. Furthermore, tumor cells targeting the pre-existing vessels of the host, a mechanism termed vascular co-option (10), has been observed to be important in brain metastasis (11) and glioma formation (12). Winkler et al (13) reported a real-time observation of glioma cells in living experimental animals. The authors concluded that perivascular glioma cells were able to move significantly faster than non-perivascular glioma cells, indicating that glioma cells utilize the perivascular space of the host as an avenue for migration. Furthermore, Farin et al (14) revealed that glioma cells were able to migrate along the perivascular space rapidly in all directions and proliferate en route when they met vascular branch points in a brain slice model. However, whether these perivascular mitotic cells form secondary tumor structures was not investigated in their study.

A growing body of evidence has suggested that invading tumor cells have distinct characteristics from those in tumor spheroids (15). For example, the invading tumor cells have a considerably lower proliferative rate than those in the tumor spheroids (15-17) and are more resistant to chemotherapy (18). Previously, Chicoine and Silbergeld (19) demonstrated that invading C6 cells isolated from the contralateral hemisphere in a rodent model were able to form tumor spheroids 
following re-implantation. On the basis of tumor recurrence in human glioblastoma, invading tumor cells dispersed in normal parenchyma may have the potential to further undergo phenotypic changes, leading to the formation of secondary tumor masses (20). Thus far, despite evidence that multiple C6 glioma models have been delineated in previous decades, how and when invading cells re-enter mitosis and form secondary tumor masses has not at present been characterized in rodent models $(6,21)$

In the present study, the features of various tumorigenic stages were re-examined, and the phenotypic alterations of implanted C6 cells in a C6 rat glioma model was investigated. In addition, the morphological and phenotypic variations of C6 cells in various regions of the tumor, specifically invading cells and cells in other regions of the tumor were characterized under high magnification. The biochemical features of the C6 cells were also characterized by immunofluorescence staining.

\section{Materials and methods}

Animals. A total of 46 adult male Sprague-Dawley (SD) rats weighing between 360 and $400 \mathrm{~g}$ were obtained from Charles River Laboratories (BioLASCO, Taipei, Taiwan). For western blot analysis, two groups of 5 rats were injected with C6 cells or phosphate-buffered saline (PBS), respectively. The remaining 36 rats were implanted with C6 cells and divided into six subgroups. Subgroups of rats were sacrificed at 3, 5, $7,9,11$ or 15 days post-implantation. All animal experimental protocols were approved by the Institutional Animal Care and Use Committee of the Chang-Gung Memorial Hospital (Chiayi, Taiwan) and performed according to the guidelines of the National Institutes of Health (Bethesda, MD, USA) for the care and use of laboratory animals.

Cell culture and brain tumor xenograft. The C6 rat glioma cell line was obtained from the American Type Culture Collection (CLL-107, Rockville, MD, USA). The cells were cultured in Dulbecco's modified Eagle's medium supplemented with $10 \%$ fetal bovine serum (Mediatech, Herndon, VA, USA) and $1 \%$ penicillin-streptomycin (Mediatech) at $37^{\circ} \mathrm{C}$ in a humidified $5 \% \mathrm{CO}_{2}$ incubator. All implantation procedures were performed according to the methods used in our previous study (22). Briefly, each rat was anesthetized with ketamine hydrochloride $(50 \mathrm{mg} / \mathrm{ml}$; Pfizer, Inc., New York, NY, USA) and then placed in a stereotactic frame (Stoelting, Wood Dale, IL, USA). Following standard aseptic preparation, the animals were implanted intracranially with $1 \times 10^{5} \mathrm{C} 6$ glioma cells. The rats were then allowed to recover from the anesthesia on a heat pad to avoid post-operative hypothermia.

Western blot analysis. The rats were decapitated following anesthesia and C6 gliomas were harvested with the aid of an operating microscope (Leica M650; Leica Microsystems $\mathrm{GmbH}$, Wetzlar, Germany). Normal rat brain tissues were also obtained from the right putamen region. Equal quantities of whole cellular protein $(50 \mu \mathrm{g})$ from the C6 gliomas, the normal brain tissues and the $\mathrm{C} 6$ cell lysates were separated on $12 \%$ sodium dodecyl sulfate-polyacrylamide gels (Sigma-Aldrich, St. Louis, MO, USA) and then transferred onto polyvinylidene difluoride membranes (Sigma-Aldrich).
The membranes were blocked with $8 \%$ non-fat dry milk for $2 \mathrm{~h}$ at room temperature and then incubated with the primary antibodies overnight at $4^{\circ} \mathrm{C}$. Antibodies to hypoxia-inducible factor 1- $\alpha$ (HIF1 $\alpha$; rabbit monoclonal; 1:200; cat. no. ab51608; Abcam, Cambridge, UK), TWIST (rabbit polyclonal; 1:200; cat. no. sc-15393; Santa Cruz Biotechnology, Inc., Santa Cruz, CA, USA), matrix metalloproteinase (MMP)-9 (monoclonal mouse; 1:400; cat. no. ab119906; Abcam), vascular endothelial growth factor (VEGF; polyclonal rabbit; 1:200; cat. no. sc-48835; Santa Cruz Biotechnology, Inc.), vascular endothelial growth factor receptor (VEGFR) 2 (monoclonal rabbit; 1:100; cat. no. sc-6251; Santa Cruz Biotechnology, Inc.), nerve growth factor (NGF; polyclonal rabbit; 1:500; cat. no. sc-548; Santa Cruz Biotechnology, Inc.), p75 neurotrophin receptor (p75; polyclonal rabbit; 1:500; cat no. ab8874; Abcam), neurotrophic tyrosine kinase receptor type 1 (TrkA; rabbit polyclonal; 1:50: cat. no. ab8871; Abcam) and $\beta$-actin (rabbit polyclonal; 1:100; cat. no. a2066; Sigma-Aldrich) were used. Following a brief rinse with Tris-buffered saline containing Tween-20, the membranes were incubated with horseradish peroxidase (HRP)-conjugated secondary antibodies. Chemiluminescence reactions were developed using SuperSignal West Pico chemiluminescent substrate (Pierce Biotechnology, Inc., Rockford, IL, USA).

Hematoxylin and eosin $(H \& E)$ staining. The rats with glioma were decapitated on the $3 \mathrm{rd}$, 5th, 7th, 9th, 11th and 15th day post-implantation ( $n=6$ in each group). The rat brains were harvested and fixed in $10 \%$ formaldehyde (Sigma-Aldrich), or preserved in optimal cutting temperature compound and stored at $-80^{\circ} \mathrm{C}$. The brain sections $(10-\mu \mathrm{m})$ were stained with $\mathrm{H} \& \mathrm{E}$ for the histological studies. The mitotic index at the center (core), periphery and tumor cluster was determined as the ratio of mitotic cells in 10 randomly selected high-power fields (magnification, $\mathrm{x} 400$ ) of brain sections from at least 3 rat gliomas harvested at 15 days post-implantation (DPI).

Immunohistochemical and immunofluorescent staining. For immunohistochemical analysis, brain sections were incubated with an antibody against MMP-2 (monoclonal mouse; 1:200; cat. no. sc-6840; Santa Cruz Biotechnology, Inc.). The sections were washed with PBS and then incubated with the HRP-conjugated secondary antibody (Superpicture HRP conjugate; Zymed, San Francisco, CA, USA). 3,3'-Diaminobenzidine tetrahydrochloride (Sigma-Aldrich) was used for chromogenic detection. For immunofluorescence analysis, the tissue sections were fixed in a 1:1 mixture of acetone-methanol for $15 \mathrm{~min}$ at room temperature and then blocked with Odyssey blocking buffer (Rockland Inc., Limerick, PA, USA). The sections were stained with antibodies against $\alpha$-smooth muscle actin ( $\alpha$-SMA; rabbit polyclonal; 1:100; cat. no. ab5694; Abcam), CD31 (mouse monoclonal; 1:50; cat. no. ab24590; Abcam), Ki67 (rabbit polyclonal; 1:200; cat. no. ab66155; Abcam), HIF1 $\alpha$ (mouse monoclonal; 1:200; Novus, Saint Charles, MO, USA), VEGF (Santa Cruz Biotechnology, Inc.), receptors for vascular endothelial growth factor (VEGFR2; goat polyclonal; 1:100; ab10972; Abcam), TWIST (rabbit polyclonal; 1:100; cat. no. ab50581; Abcam), Vimentin (mouse monoclonal; 1:200; cat. no. sc-6260; Santa Cruz Biotechnology, Inc.), p75 (rabbit 
polyclonal; 1:50; cat. no. ab38335; Abcam) and TrkA (rabbit polyclonal; $1: 50$; cat. no. ab8871; Abcam). The sections were incubated with secondary antibodies (Invitrogen Life Technologies) for $1 \mathrm{~h}$ at room temperature. The nuclei were stained with DAPI (Invitrogen Life Technologies) for $30 \mathrm{sec}$ at $37^{\circ} \mathrm{C}$. The expression levels of other proteins detected by immunohistochemical or immunofluorescence staining were evaluated using a semi-quantitative method (23). The scoring system was defined as follows:,$-<1 \%$ positive cells;,$+ 2-25 \%$ positive cells;,$++ 26-50 \%$ positive cells;,$+++ 51-75 \%$ positive cells;,$++++>75 \%$ positive cells. The Ki67 index at the center, periphery and tumor cluster were calculated as the ratio of Ki67-positive cells in 10 randomly selected high-power fields (magnification, $\mathrm{x} 400$ ) of brain sections from at least three rat gliomas harvested at $15 \mathrm{DPI}$.

Experimental analysis. Images of $\mathrm{H} \& \mathrm{E}$, immunohistochemical and immunofluorescence staining were captured and analyzed using a CCD camera (Eclipse E600, Nikon, Tokyo, Japan) and Image ProPlus 3.0 software (Media Cybernetics, Sarasota, FL, USA).

Statistical analysis. The mitotic rate and $\mathrm{Ki} 67$ index were compared by performing a one-way analysis of variance. $\mathrm{P}<0.05$ was considered to indicate a statistically significant difference. All statistical tests were two-tailed and performed using SPSS, version 12.0 (SPSS, Inc., Chicago, IL, USA).

\section{Results}

Formation of C6 glioma in SD rats and gene expression in C6-derived tumors. C6 cells $\left(1 \times 10^{5}\right)$ were stereotactically injected into the right putamen region of male adult SD rats to establish a C6 glioma model. The SD rats implanted with C6 cells consistently succumbed to glioma at 15-18 DPI in our established system (22). As the tumor grew, the C6 glioma protruded from the surface of rat brain with a whitish, soft, and well-circumscribed nodular appearance (Fig. 1A). From a cross-sectional view, the tumor mass formed at 15 DPI, and exhibited small hemorrhagic spots, distorted structures and necrotic portions (Fig. 1B).

To examine the differential gene expression among normal brains, C6 gliomas and the cultured C6 cells, several known tumor-associated genes were detected in these samples. As shown in Fig. 1C, the samples from the mature C6 gliomas exhibited a higher expression of HIF1 $\alpha$, TWIST (23), MMP-9, VEGF, VEGFR, NGF (24) and TrkA (25), when compared with those from normal brain tissue and the cultured C6 cells. These results indicated the successful establishment of a C6 glioma model and implied that the neural micro-environment in SD rats is able to further modulate the expression of specific tumor-associated genes in C6 cells.

Formation of the primary tumor spheroids and neovascularization during the early stages of tumorigenesis. To further characterize the morphological or functional changes of tumor cells during the progression of C6 glioma, brain specimens were harvested during the period between 3-15 DPI. At 3 DPI, C6 cells initially formed a small tumor spheroid at the site of injection (Fig. 2A). The C6 cells were densely packed
A

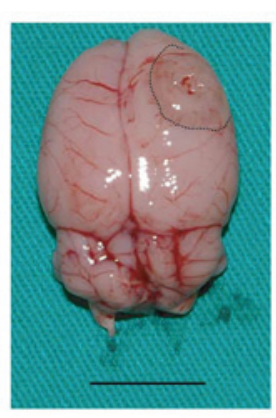

B
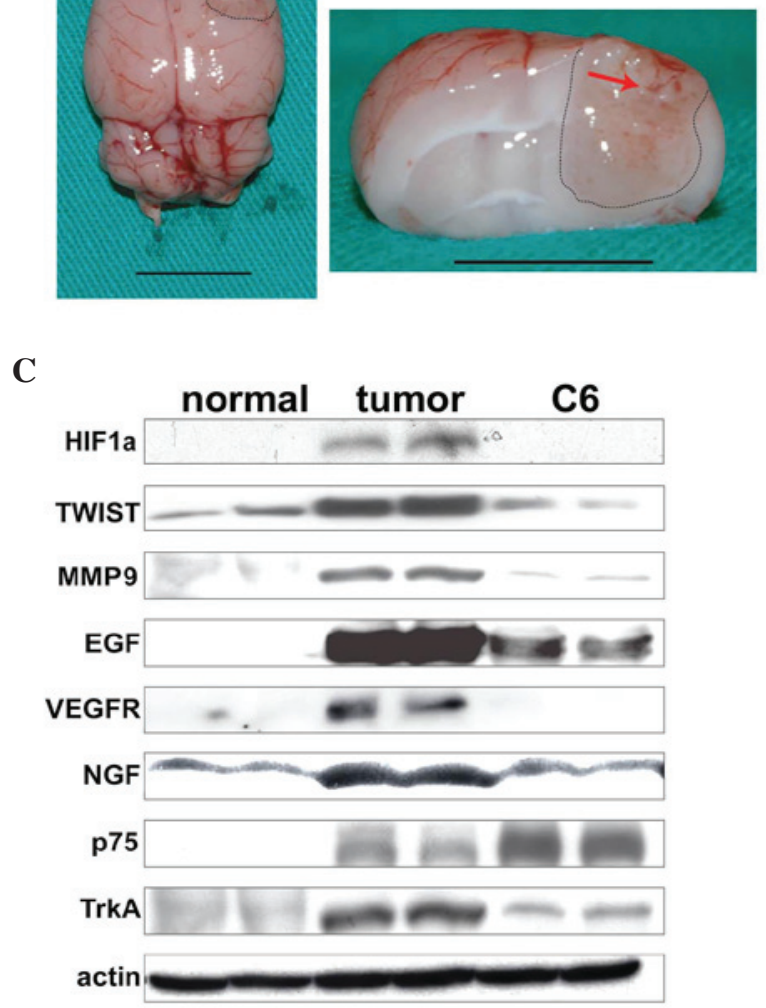

Figure 1. Relative expression level of tumor-associated genes in normal brain tissues (normal), C6 glioma tissues (tumor) and cultured C6 cells (C6). (A) Superior view of a rat brain with C6 glioma (scale bar, $1 \mathrm{~cm}$ ). (B) Cross section of a rat brain with C6 glioma. The tumor formed at the right hemisphere revealed a blurred margin (black dotted line) with petechial hemorrhage in the central region (red arrow; scale bar, $1 \mathrm{~cm}$ ). (C) Expression of tumor-associated genes in normal brain tissues (normal), C6 glioma tissues (tumor) and cultured C6 cells (C6). HIF1 $\alpha$, hypoxia-inducible factor 1- $\alpha$, MMP, matrix metalloproteinase; VEGF, vascular endothelial growth factor; VEGFR, vascular endothelial growth factor receptor; NGF, nerve growth factor; p75, p75 neurotrophin receptor; TrkA, neurotrophic tyrosine kinase receptor type 1 .

in the tumor spheroid with a demarcated border. These tumor cells in the spheroid were round or oval and contained a pleomorphic nucleus (Fig. 2B). A minor hemorrhage caused by the injection of the C6 cells was noted on the wall of the injection tract.

At 5 DPI, active neovascularization was observed in the tumor and the parenchyma near the tumor margin (Fig. 2C and D). Features at this stage included the presence of actively dividing cells (Fig. 2E and F) and the formation of new blood vessels at the tumor margin.

Tumor cell invasion at 7-9 DPI. At 7-9 DPI, tumor cells underwent prolific spreading and invasion, which resulted in the formation of a diffuse and irregular front zone (thin region outside visual boundary layer of tumor) surrounding the tumor (Fig. 3A and 3B). The invading tumor cells were able to migrate far from the tumor margin and aggregate along the host blood vessels outside the tumor periphery (Fig. 3C). Under a high magnification view (magnification, $\mathrm{x} 1,000)$, active mitosis of the perivascular tumor cells was 
A

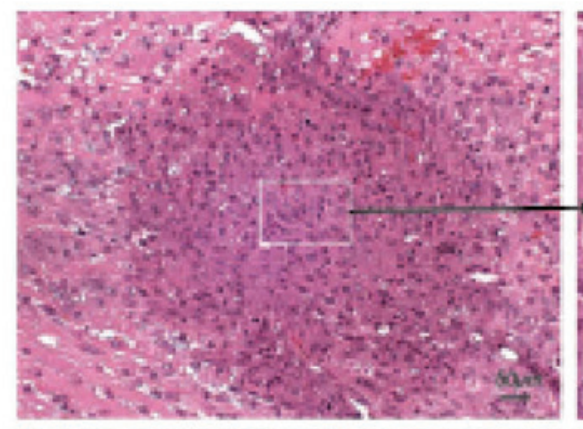

C

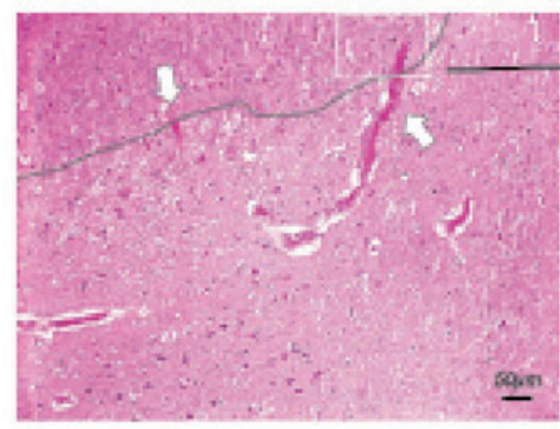

$\mathbf{E}$

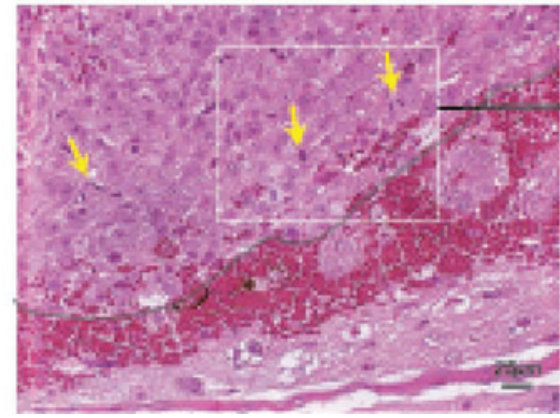

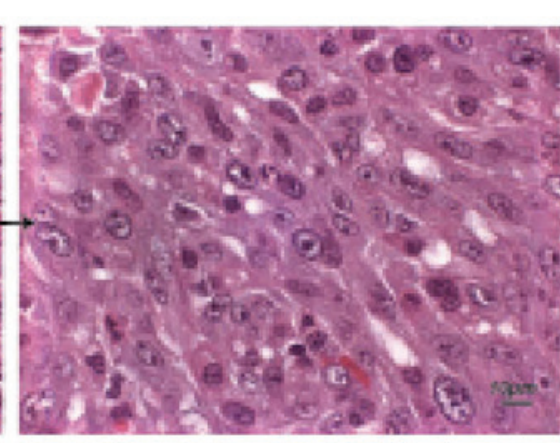

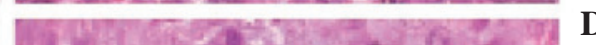

Figure 2. Morphological features occurring in early stages (3-5 DPI) of a C6 glioma. (A) Small tumor spheroids were detected at 3 DPI. The paraffin-embedded section was stained with H\&E (scale bar, $50 \mu \mathrm{m}$ ). (B) C6 cells were densely compacted at the injection site and exhibited nuclear pleomorphism at this stage (H\&E; scale bar, $10 \mu \mathrm{m}$ ). (C) Prominent neovascularization was observed at the tumor margin (black dash line) at 5 DPI (H\&E; scale bar, $50 \mu \mathrm{m}$ ). The white arrows indicate blood vessels. (D) Magnified view (H\&E; scale bar, $10 \mu \mathrm{m}$ ) of the white dashed rectangle from (C). White arrows indicate blood vessels and yellow arrows indicate mitotic cells. (E) Tumor margins remained smooth (black dash line, tumor margin; yellow arrow, cell mitosis) at 5 DPI. (H\&E; scale bar, $25 \mu \mathrm{m}$ ). (F) Magnified view (H\&E; scale bar, $10 \mu \mathrm{m}$ ) of the white dashed rectangle of panel (E). Yellow arrows indicate mitotic cells. DPI, days post-implantation; H\&E, hematoxylin and eosin.

detected (Fig. 3D, yellow arrow). It was noted that tumor cells at different locations exhibited different morphological characteristics at this stage. Tumor cells within the tumor core were generally round to oval, and densely packed (Fig. 3E). Mitotic cells could be observed in the tumor core (Fig. 3F, yellow arrow). By contrast, the tumor cells near the tumor margin (periphery) exhibited a larger cell size and larger nuclei with irregularly marginated chromatin (Fig. 3G). Outside the tumor margin, certain migrating tumor cells were clustered around the blood vessels of the host (Fig. 3H). These perivascular tumor cells exhibited a significant mitotic count, and partially corresponded with the intensive staining of H\&E similar to those in the center of the tumor (Fig. 3F, yellow arrow).

Occurrence of tumor necrosis and formation of tumor cell multilayer clusters at 11-15 DPI. At 11-15 DPI, the C6 glioma revealed typical features of a malignant glioma, including hemorrhage, necrosis (Fig. 4A), and pseudopalisades (26) (Fig. 4B). Similar to the rat gliomas at 7-9 DPI, the morphology of tumor cells near the tumor margin (Fig. 4C) was significantly different from the tumor cells in the tumor core (Fig. 4D). Notably, tumor cell multi-layer clusters distant from the primary tumor mass could be detected at this stage (Fig. 4E and F). Serial sample sections were examined to exclude the possibility that these clusters were extensions of the primary tumor mass. Specifically, all these multi-layer cell clusters were formed surrounding a host blood vessel, which was confirmed by staining with $\alpha$-SMA (Fig. 4G) and CD31 (Fig. 4H). Based on the diameter $(20-40 \mu \mathrm{m})$ of the blood vessels detected by $\alpha$-SMA staining (Fig. 4G), it was suggested that these blood vessels may represent small to medium size arterioles $(27,28)$.

Differential cell proliferation rate in different tumor areas. To investigate the proliferative rate of tumor cells in the tumor core, tumor periphery and tumor cell clusters, the mitotic index and Ki67 index were determined using H\&E and Ki67 staining of tumor sections. Although there is no strict border between the tumor core and tumor periphery, the tumor core 

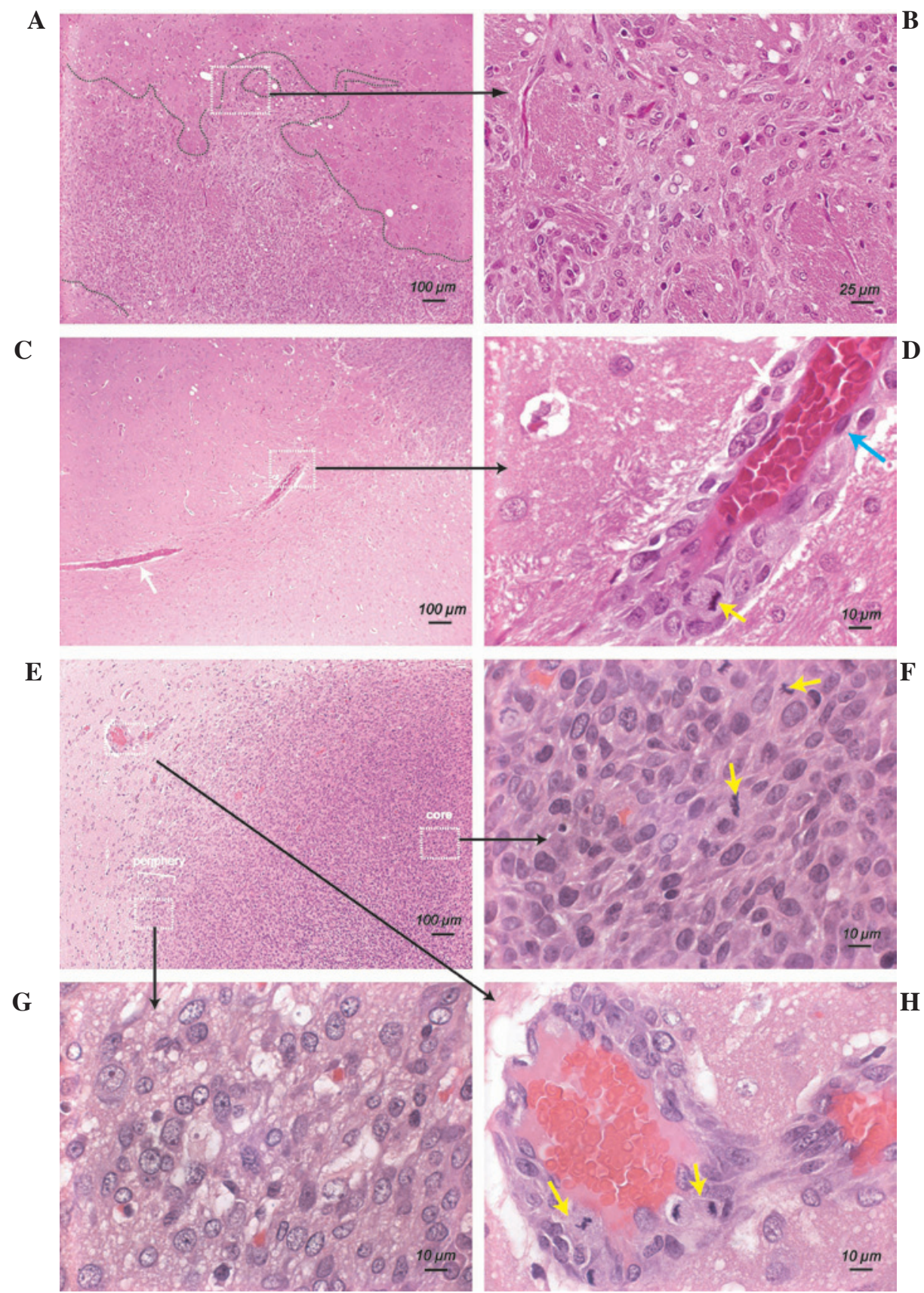

Figure 3. Active invasion of C6 cells at 7-9 DPI. (A) Formation of unusual tumor margins following extensive invasion of tumor cells into normal parenchyma (H\&E; scale bar, $100 \mu \mathrm{m}$ ) (B) Magnified view (H\&E; scale bar, $25 \mu \mathrm{m}$ ) of the white rectangle from (A). Tumor cells grew in a disorganized manner. (C) Migration of tumor cells along an adjacent blood vessel (white rectangle) (H\&E; scale bar, $100 \mu \mathrm{m}$ ). (D) Microscopic view (H\&E; scale bar, $10 \mu \mathrm{m})$ of the white rectangle from (C). Ongoing mitosis (yellow arrow) was observed in perivascular tumor cells (H\&E; scale bar, $10 \mu \mathrm{m})$. The blue arrow indicates an endothelial cell. (E) Photomicrograph of tumor core and tumor periphery (H\&E; scale bar, $100 \mu \mathrm{m}$ ). (F) High magnification view of the right rectangle (H\&E; scale bar, $10 \mu \mathrm{m}$ ) from (E). The yellow arrows indicate cells undergoing mitosis. (G) Magnified view of the bottom rectangle (H\&E; scale bar, $10 \mu \mathrm{m})$ from (E). (H) Magnified view of the left rectangle (H\&E; scale bar, $10 \mu \mathrm{m}$ ) from (E). Mitotic tumor cells (yellow arrow) were present in the perivascular space of a blood vessel. H\&E, hematoxylin and eosin; DPI, days post-implantation.

was defined as the region in the central section of the tumor or just next to regions of tumor necrosis, while the tumor periphery was defined as region in the extreme perimeter of the tumor margin.

As shown in Fig. 5, the average mitotic index was $2.39 \pm 0.7 \%$ in the tumor core, $1.0 \pm 0.35 \%$ in the tumor periphery and $5.84 \pm 1.5 \%$ in the secondary tumor clusters

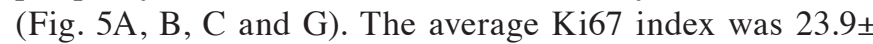
$4.7 \%$ in the tumor core, $9.6 \pm 1.7 \%$ in the tumor periphery and $39.5 \pm 6.2 \%$ in the newly formed clusters (Fig. 5D, E, F and H).
These results indicate that tumor cells in the secondary tumor clusters have the highest cell proliferation rate, while tumor cells proliferate poorly in the tumor periphery.

Differential expression of tumor-associated genes in different areas of C6 glioma. To characterize gene expression patterns in different areas of a mature glioma at 11-15 DPI, the expression of tumor-associated genes, including MMP-2, HIF $1 \alpha$, VEGF, VEGFR2, TWIST, Vimentin, p75 (29) and TrkA, were analyzed using immunohistochemistry or immunofluorescence 


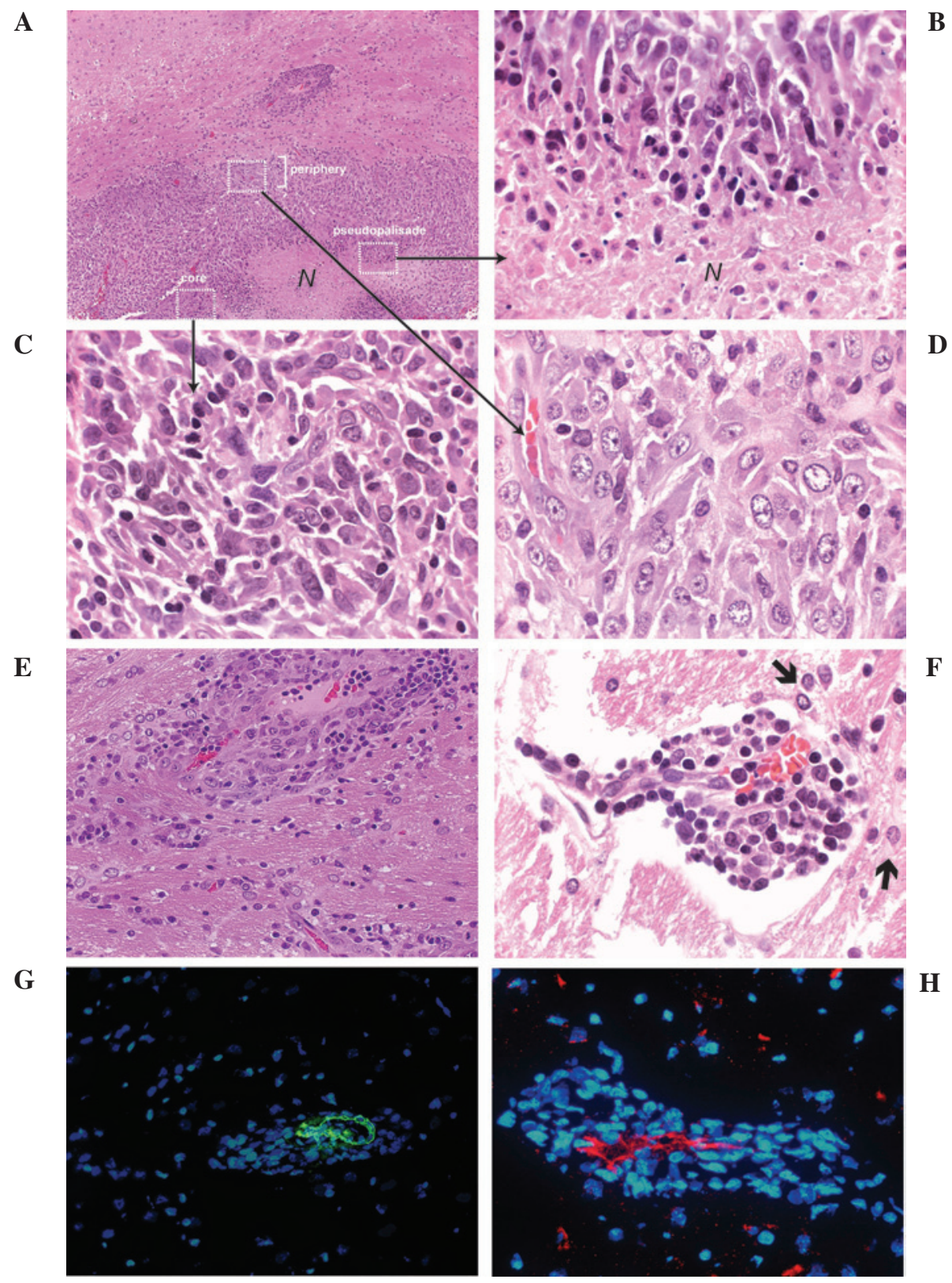

Figure 4. Occurrence of central necrosis and formation of highly proliferative tumor cell clusters at 11-15 DPI. (A) Malignant C6 glioma at 11 DPI (H\&E; scale bar, $100 \mu \mathrm{m})$. N represents necrosis, C indicates a small tumor cell cluster and white rectangles represent the region of the tumor core, periphery and pseudopalisades. (B) At the perinecrotic region, pseudopalisading C6 cells with condensed nuclei and an elongated cytoplasm were observed in rows (H\&E; scale bar, $10 \mu \mathrm{m}$ ). (C) Tumor cells carrying compact hyperchromatic nuclei were detected in the tumor core (H\&E; scale bar, $10 \mu \mathrm{m}$ ). (D) Larger, loosely arranged tumor cells were identified at the periphery of the tumor. (H\&E; scale bar, $10 \mu \mathrm{m}$ ). (E and F) Multi-layer tumor cell clusters were formed around small blood vessels. As noted, the endothelium of the blood vessel exhibited regression in the distal section (H\&E; scale bar, $10 \mu \mathrm{m}$ ). Black arrow indicates a migrating cell. (G and H) Immunofluorescence staining with anti- $\alpha$-smooth muscle actin and anti-CD31 antibodies (Scale bar, $25 \mu \mathrm{m}$ ). H\&E, hematoxylin and eosin; DPI, days post-implantation.

as summarized in Table I. As shown in Fig. 6, MMP-2 expression was predominantly detected in the periphery of the glioma. By contrast, HIF1 $\alpha$ was highly expressed in the tumor core, particularly in the perinecrotic region. Notably, HIF1 $\alpha$, VEGF and VEGFR2 were abundantly expressed in the tumor core and the tumor clusters, but not in the periphery of the glioma. Despite different tumor cell densities in different areas of the glioma, the positive staining for TWIST, Vimentin and TrkA appeared to be distributed evenly in the different tumor areas. In addition, p75 was abundantly expressed in the secondary tumor clusters when compared with the tumor core or periphery.

\section{Discussion}

The C6 glioma model is a well-established malignant brain tumor model, which shares a number of histopathological features with human GBM (30). Although the cytological features of C6 glioma progression have been described in previous decades, the potential phenotypic switch of C6 cells in rodent hosts remains to be elucidated. Thus, re-examination of the phenotypic changes of C6 cells in the growing tumors remains important to verify or support certain newly developing hypotheses. In the present study, it was demonstrated that a number of tumor-associated genes in C6 cells may be further 

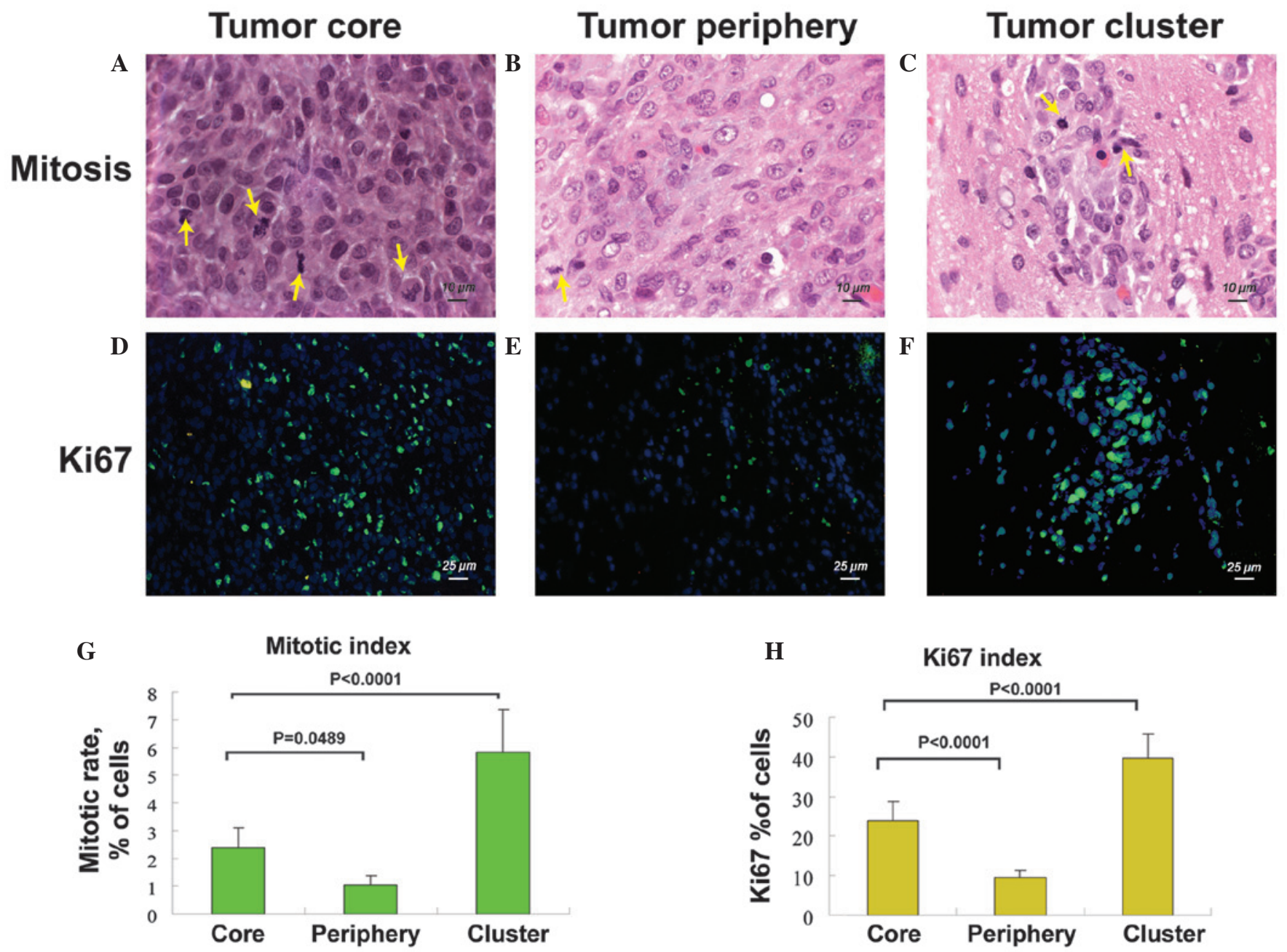

Figure 5. Differential proliferation of C6 cells in different regions of a mature glioma. Hematoxylin and eosin, and Ki67 staining of tumor sections (11-15 DPI) were used to determine tumor cell proliferation. Representative images of mitotic cells (yellow arrows) and Ki67-positive cells (green) present in the tumor core (A and D), tumor periphery (B and E) and tumor clusters (C and F) are shown. (G) Percentage of mitotic cells in the tumor core, tumor periphery and tumor clusters, $\mathrm{P}<0.0001$ or $\mathrm{P}<0.05$. (H) Percentage of Ki67-positive tumor cells in the tumor core, tumor periphery and tumor clusters, $\mathrm{P}<0.0001$.

upregulated following implantation (Fig. 1C), suggesting that the neural micro-environment in SD rats stimulates the expression of these tumor-associated genes. These genes include HIF-1 $\alpha$, TWIST, MMP-9, VEGF, VEGFR, NGF, and TrkA. Additionally, the present results provided evidence of the phenotypic changes of invading tumor cells and the formation of secondary tumor clusters during the late tumorigenic stages.

In the present study, significant morphological and phenotypic differences were identified between the tumor cells in the tumor core and those in the tumor periphery during tumor growth. Tumor cells with densely stained nuclei were closely packed in the tumor core, in which mitotic cells were frequently identified (Fig. 3F). By contrast, invading tumor cells at the periphery were loosely arranged and exhibited marginated chromatin (Fig. 3G). As suggested previously, the morphological and phenotypic differences between invading tumor cells and those in the tumor core may be due to the particular local environment (16). Tumor cells responding to different supplies of nutrients, oxygen or other regulatory factors may produce differential characteristics during the development of a tumor (31). In addition to the morphological
Table I. Spatial expression profiles of MMP-2, HIF1 $\alpha$, VEGF, VEGFR2, TWIST, Vimentin, p75 and TrkA in C6 glioma.

\begin{tabular}{lccc}
\hline Gene & $\begin{array}{c}\text { Tumor } \\
\text { periphery }\end{array}$ & $\begin{array}{c}\text { Tumor } \\
\text { core }\end{array}$ & Cluster \\
\hline MMP-2 & +++ & + & + \\
HIF1 $\alpha$ & -- & +++ & ++ \\
VEGF & + & +++ & +++ \\
VEGFR & + & +++ & +++ \\
TWIST & ++ & +++ & +++ \\
p75 & + & ++ & ++++ \\
Vimentin & ++ & +++ & +++ \\
trkA & ++ & +++ & +++ \\
\hline
\end{tabular}

The score was defined in three randomly selected high power fields (magnification, $\mathrm{x} 400$ ) as follows:,$-<1 \%$ positive cells;,$+ 2-25 \%$ positive cells;,$++ 26-50 \%$ positive cells;,$+++ 51-75 \%$ positive cells; ,$++++>75 \%$ positive cells. HIF1 $\alpha$, hypoxia-inducible factor $1-\alpha$, MMP, matrix metalloproteinase; VEGF, vascular endothelial growth factor; VEGFR, vascular endothelial growth factor receptor; NGF, nerve growth factor; p75, p75 neurotrophin receptor; TrkA, neurotrophic tyrosine kinase receptor type 1 . 




Figure 6. Spatial expression profiles of MMP-2, HIF1 $\alpha$, VEGF, VEGFR2, TWIST, Vimentin, p75 and TrkA in C6 glioma. The expression of the indicated tumor-associated genes in tissue sections of $\mathrm{C} 6$ glioma was detected by immunohistochemistry or immunofluorescence. The white dotted line indicates the tumor margin and T indicates the tumor. HIF1 $\alpha$, hypoxia-inducible factor 1- $\alpha$, MMP, matrix metalloproteinase; VEGF, vascular endothelial growth factor; VEGFR, vascular endothelial growth factor receptor; NGF, nerve growth factor; p75, p75 neurotrophin receptor; TrkA, neurotrophic tyrosine kinase receptor type 1 . 
changes, it was also demonstrated that invading tumor cells have a relatively lower proliferation rate (Fig. 5) than those in the tumor core and may be more resistant to apoptosis $(15,18)$, which contributes to their invasive nature $(17,32)$. These observations are consistent with the previous studies concerning the dichotomy of cancer cell migration and invasion $(16,17)$. Notably, when the invading tumor cells dock at blood vessels and form tumor clusters outside the primary tumor mass, these tumor cells exhibited a high cell proliferation rate. This suggests that invading cells may have the potential to undergo another phenotypic change and continue to grow into a secondary tumor mass under appropriate conditions.

The infiltration of tumor cells has been known to form the structures termed 'secondary structures of Scherer' in human GBM (33). The infiltration of tumor cells is also regarded as the origin of multifocal or multicentric human glioblastomas and is associated with a poor prognosis (20). However, it has rarely been investigated when or how the invading glioma cells re-enter mitosis and form the secondary tumors in animal models $(6,21)$. Previously, Zhao et al (34) established a xenograft mouse model using an MMP-9-expressing U1242 MG glioma cell line revealing an extensive invasion pattern. Large numbers of individual tumor cells and cell clusters outside the implanted site were observed in their model, and silencing of MMP-9 expression in the U1242 MG cells significantly inhibited the motility of tumor cells and the formation of tumor clusters. By contrast, treatment of the xenograft rodent model with anti-VEGF antibodies was able to reduce tumor blood flow and volume, which increased hypoxia in the tumor core and resulted in the enhancement of tumor cell invasion $(35,36)$. Furthermore, the invading tumor cells in these two models (MMP overexpression and anti-VEGF treatment) exhibit significant perivascular invasion and formation of multiple tumor clusters by co-option of the host vasculature at the tumor periphery. Although cell clusters or satellite tumors may be detected in these specific models, it remains to be elucidated whether the formation of cell clusters or satellite tumors is a common event during glioma development in rodent models. Furthermore, the characteristics of these cell clusters or satellite tumors remain elusive. In the present study, it was demonstrated that the formation of cell clusters or satellite tumors may be observed at the later stages of the C6 glioma model and it may represent a common event in rodent models. Evidence was also provided to demonstrate that tumor cell clusters are formed by vascular co-option as proposed by Holash et al (12). On the basis of their hypothesis, the tumor formation (of the primary tumor) undergoes blood vessel co-option, blood vessel regression and tumor growth. In addition, the growth of the secondary tumor clusters appears to follow a similar path (Figs. 3 and 4).

Several specific features have been identified for these tumor cell clusters, including multilayers ( $>3$ layers) of tumor cells surrounding a blood vessel (20-40 $\mu \mathrm{m})$ up to several millimeters away from the primary tumor mass, a high proliferation rate comparable to that in the tumor core, and similar gene expression patterns to that in the tumor core (Fig. 6). Notably, not all microvessels near the primary tumor were observed to support the formation of tumor cell clusters. Therefore, tumor cell clusters may only be formed when the invading cells identify a hospitable perivascular space as suggested by the 'seed and soil' hypothesis $(37,38)$. As the small tumor cell clusters may potentially be the origin of the secondary tumors, the characterization of these cell clusters may be useful for elucidating the micro-environmental regulation of tumor-host interaction.

Immunohistochemical and immunofluorescent staining were performed to compare the expression of specific tumor-associated markers between different tumor regions, including the tumor core, tumor periphery and secondary tumor clusters. The present findings indicated that tumor cells in the tumor clusters express similar biomarkers to those in the tumor core, but not to those in the tumor periphery. The tumor cells in the secondary tumor clusters, although derived from the aggregation and mitosis of perivascular invading cells, exhibited higher expression levels of HIF1 $\alpha$, VEGF and VEGFR, but not MMP-2, than the migrating tumor cells from the tumor periphery (Table I). These findings imply that the secondary tumor clusters possess an enhanced capacity to induce angiogenesis. A significantly higher expression of p75 was also observed in tumor clusters as compared with that in the tumor periphery or in the tumor core, suggesting p75 may be critical in tumor re-initiation at different sites.

In conclusion, the progression of C6 glioma in SD rats was re-characterized and evidence for the existence of the perivascular tumor clusters surrounding the tumor margin was provided, which may serve as the origin for the secondary tumors. These findings clearly suggest that the phenotypic switch of invasive tumor cells and the specific surrounding microenvironment contribute to the initiation of the secondary tumor formation.

\section{Acknowledgements}

The present study was supported by grants from the Chang-Gung Memorial Hospital (grant nos. CMRP680401, CMRPG680402 and CMRPD680403). The authors would like to express their sincere appreciation to Miss I-Gin Lin for her extensive technical assistance.

\section{References}

1. Stupp R,Mason WP, van den Bent MJ,Weller M,FisherB,Taphoorn MJ, Belanger K, Brandes AA, Marosi C, Bogdahn U, et al; European Organisation for Research and Treatment of Cancer Brain Tumor and Radiotherapy Groups; National Cancer Institute of Canada Clinical Trials Group: Radiotherapy plus concomitant and adjuvant temozolomide for glioblastoma. N Engl J Med 352: 987-996, 2005.

2. Taylor LP: Diagnosis, treatment, and prognosis of glioma: Five new things. Neurology 75 (Suppl 1): S28-S32, 2010.

3. Nagano N, Sasaki H, Aoyagi M and Hirakawa K: Invasion of experimental rat brain tumor: Early morphological changes following microinjection of C6 glioma cells. Acta Neuropathol 86: 117-125, 1993.

4. Vajkoczy P, Farhadi M, Gaumann A, Heidenreich R, Erber R, Wunder A, Tonn JC, Menger MD and Breier G: Microtumor growth initiates angiogenic sprouting with simultaneous expression of VEGF, VEGF receptor-2, and angiopoietin-2. J Clin Invest 109: 777-785, 2002.

5. Karmakar S, Olive MF, Banik NL and Ray SK: Intracranial stereotaxic cannulation for development of orthotopic glioblastoma allograft in Sprague-Dawley rats and histoimmunopathological characterization of the brain tumor. Neurochem Res 32: 2235-2242, 2007.

6. Vince GH, Bendszus M, Schweitzer T, Goldbrunner RH, Hildebrandt S, Tilgner J, Klein R, Solymosi L, Christian Tonn J and Roosen K: Spontaneous regression of experimental gliomas - an immunohistochemical and MRI study of the C6 glioma spheroid implantation model. Exp Neurol 190: 478-485, 2004. 
7. Sibenaller ZA, Etame AB, Ali MM, Barua M, Braun TA, Casavant TL and Ryken TC: Genetic characterization of commonly used glioma cell lines in the rat animal model system. Neurosurg Focus 19: E1, 2005

8. Bernstein JJ, Laws ER Jr, Levine KV, Wood LR, Tadvalkar G and Goldberg WJ: C6 glioma-astrocytoma cell and fetal astrocyte migration into artificial basement membrane: A permissive substrate for neural tumors but not fetal astrocytes. Neurosurgery 28: 652-658, 1991.

9. Pedersen PH, Marienhagen K, Mørk S and Bjerkvig R: Migratory pattern of fetal rat brain cells and human glioma cells in the adult rat brain. Cancer Res 53: 5158-5165, 1993.

10. Carbonell WS, Ansorge O, Sibson N and Muschel R: The vascular basement membrane as 'soil' in brain metastasis. PLoS One 4: e5857, 2009.

11. Kienast Y, von Baumgarten L, Fuhrmann M, Klinkert WE, Goldbrunner R, Herms J and Winkler F: Real-time imaging reveals the single steps of brain metastasis formation. Nat Med 16: 116-122, 2010

12. Holash J, Maisonpierre PC, Compton D, Boland P, Alexander CR, Zagzag D, Yancopoulos GD and Wiegand SJ: Vessel cooption, regression, and growth in tumors mediated by angiopoietins and VEGF. Science 284: 1994-1998, 1999.

13. Winkler F, Kienast Y, Fuhrmann M, Von Baumgarten L, Burgold S, Mitteregger G, Kretzschmar H and Herms J: Imaging glioma cell invasion in vivo reveals mechanisms of dissemination and peritumoral angiogenesis. Glia 57: 1306-1315, 2009.

14. Farin A, Suzuki SO, Weiker M, Goldman JE, Bruce JN and Canoll P: Transplanted glioma cells migrate and proliferate on host brain vasculature: A dynamic analysis. Glia 53: 799-808, 2006

15. Giese A, Bjerkvig R, Berens ME and Westphal M: Cost of migration: Invasion of malignant gliomas and implications for treatment. J Clin Oncol 21: 1624-1636, 2003.

16. Mariani L, Beaudry C, McDonough WS, Hoelzinger DB Kaczmarek E, Ponce F, Coons SW, Giese A, Seiler RW and Berens ME: Death-associated protein 3 (Dap-3) is overexpressed in invasive glioblastoma cells in vivo and in glioma cell lines with induced motility phenotype in vitro. Clin Cancer Res 7: 2480-2489, 2001.

17. Giese A, Loo MA, Tran N, Haskett D, Coons SW and Berens ME: Dichotomy of astrocytoma migration and proliferation. Int J Cancer 67: 275-282, 1996.

18. Saito R, Bringas J, Mirek H, Berger MS and Bankiewicz KS: Invasive phenotype observed in 1,3-bis(2-chloroethyl)-1-nitrosourea-resistant sublines of 9L rat glioma cells: A tumor model mimicking a recurrent malignant glioma. J Neurosurg 101: 826-831, 2004

19. Chicoine MR and Silbergeld DL: Invading C6 glioma cells maintaining tumorigenicity. J Neurosurg 83: 665-671, 1995.

20. Hassaneen W, Levine NB, Suki D, Salaskar AL, de Moura Lima A, McCutcheon IE, Prabhu SS, Lang FF, DeMonte F, Rao G, et al Multiple craniotomies in the management of multifocal and multicentric glioblastoma. Clinical article. J Neurosurg 114: 576-584, 2011

21. Grobben B, De Deyn PP and Slegers H: Rat C6 glioma as experimental model system for the study of glioblastoma growth and invasion. Cell Tissue Res 310: 257-270, 2002.

22. Wang TC, Hsiao IT, Cheng YK, Wey SP, Yen TC and Lin KJ: Noninvasive monitoring of tumor growth in a rat glioma model Comparison between neurological assessment and animal imaging. J Neurooncol 104: 669-678, 2011.
23. Mikheeva SA, Mikheev AM, Petit A, Beyer R, Oxford RG, Khorasani L, Maxwell JP, Glackin CA, Wakimoto H, González-Herrero I, et al: TWIST1 promotes invasion through mesenchymal change in human glioblastoma. Mol Cancer 9: 194, 2010

24. Walsh EM, Kim R, Del Valle L, Weaver M, Sheffield J, Lazarovici $\mathrm{P}$ and Marcinkiewicz C: Importance of interaction between nerve growth factor and $\alpha 9 \beta 1$ integrin in glial tumor angiogenesis. Neuro-oncol 14: 890-901, 2012.

25. Assimakopoulou M, Kondyli M, Gatzounis G, Maraziotis T and Varakis J: Neurotrophin receptors expression and JNK pathway activation in human astrocytomas. BMC Cancer 7: 202, 2007.

26. Brat DJ and Van Meir EG: Vaso-occlusive and prothrombotic mechanisms associated with tumor hypoxia, necrosis, and accelerated growth in glioblastoma. Lab Invest 84: 397-405, 2004.

27. McCaslin AF, Chen BR, Radosevich AJ, Cauli B and Hillman EM: In vivo 3D morphology of astrocyte-vasculature interactions in the somatosensory cortex: Implications for neurovascular coupling. J Cereb Blood Flow Metab 31: 795-806, 2011.

28. Seylaz J, Charbonné R, Nanri K, Von Euw D, Borredon J, Kacem K, Méric P and Pinard E: Dynamic in vivo measurement of erythrocyte velocity and flow in capillaries and of microvessel diameter in the rat brain by confocal laser microscopy. J Cereb Blood Flow Metab 19: 863-870, 1999.

29. Johnston AL, Lun X, Rahn JJ, Liacini A, Wang L, Hamilton MG, Parney IF, Hempstead BL, Robbins SM, Forsyth PA, et al: The p75 neurotrophin receptor is a central regulator of glioma invasion. PLoS Biol 5: e212, 2007.

30. Jacobs VL, Valdes PA, Hickey WF and De Leo JA: Current review of in vivo GBM rodent models: Emphasis on the CNS-1 tumour model. ASN Neuro 3: e00063, 2011.

31. Gorin F, Harley W, Schnier J, Lyeth B and Jue T: Perinecrotic glioma proliferation and metabolic profile within an intracerebral tumor xenograft. Acta Neuropathol 107: 235-244, 2004.

32. Hoelzinger DB, Mariani L, Weis J, Woyke T, Berens TJ, McDonough WS, Sloan A, Coons SW and Berens ME: Gene expression profile of glioblastoma multiforme invasive phenotype points to new therapeutic targets. Neoplasia 7: 7-16, 2005

33. Zagzag D, Lukyanov Y, Lan L, Ali MA, Esencay M, Mendez O, Yee H, Voura EB and Newcomb EW: Hypoxia-inducible factor 1 and VEGF upregulate CXCR4 in glioblastoma: Implications for angiogenesis and glioma cell invasion. Lab Invest 86 1221-1232, 2006

34. Zhao Y, Xiao A, diPierro CG, Carpenter JE, Abdel-Fattah R, Redpath GT, Lopes MB and Hussaini IM: An extensive invasive intracranial human glioblastoma xenograft model: Role of high level matrix metalloproteinase 9. Am J Pathol 176: 3032-3049, 2010.

35. Rubenstein JL, Kim J, Ozawa T, Zhang M, Westphal M, Deen DF and Shuman MA: Anti-VEGF antibody treatment of glioblastoma prolongs survival but results in increased vascular cooption. Neoplasia 2: 306-314, 2000.

36. Keunen O, Johansson M, Oudin A, Sanzey M, Rahim SA, Fack F Thorsen F, Taxt T, Bartos M, Jirik R, et al: Anti-VEGF treatment reduces blood supply and increases tumor cell invasion in glioblastoma. Proc Natl Acad Sci USA 108: 3749-3754, 2011.

37. Fidler IJ: The pathogenesis of cancer metastasis: The 'seed and soil' hypothesis revisited. Nat Rev Cancer 3: 453-458, 2003.

38. Fidler IJ and Poste G: The 'seed and soil' hypothesis revisited. Lancet Oncol 9: 808, 2008. 